\title{
CRÉATION ET SIMULATION DE JETS HYPERVELOCES
}

\author{
J.P. LEYRAT* ${ }^{*}$ E. CHARVET* ${ }^{*}$, M. MACE* et H.C. PUJOL S $^{*}$ \\ "Centre d'Etudes de Limeil-Valenton, F-94195 \\ Villeneuve-Saint-Georges Cedex, France \\ ${ }^{*}$ CEA-CESTA, F-94195 Villeneuve-Saint-Georges Cedex, France
}

\begin{abstract}
Résumé - Nous proposons un nouveau concept pour obtenir des jets métalliques trés rapides (dont la vitesse peut atteindre ou dépasser $20 \mathrm{~km} / \mathrm{s}$ ). On utilise la détonation engendrée dans un cylindre d'explosif pour initier une onde de compression sur la face arrière d'une cible métallique; le jet est crée en face avant, dans une cavité cylindrique. Un grand nombre d'expériences ont permis de paramétrer l'influence de la nature du matériau (cuivre ou alliage d'aluminium AU4G), et de la géométrie de la cavité. Des calculs numériques de simulation ont été effectués avec PLEXUS (code éléments finis 2D ou 3D) et CEL (code 2D différences finies); ils ont permis de comprendre le processus de création et de propagation du jet, et de restituer correctement les observations expérimentales (forme et vitesse du jet).
\end{abstract}

\begin{abstract}
We propose a new concept in order to produce very fast metallic jets (which speed could exceed 20 $\mathrm{km} / \mathrm{s}$ ). It consists in using the detonation wave of an explosive cylinder to initiate a high compression on the rear face of a metallic target; the jet is created on the front surface, in a cylindrical cavity. A lot of experiments were performed, in which we tested two different metals (copper and aluminum alloy AU4G) and various geometries of cavity. Numerical calculations with PLEXUS (finite elements $2 D$ or $3 D$ code) and CEL (finite differences $2 D$ code) were used to understand the phenomenology of the creation and propagation of these jets ; the correlation with the experimental results (about the shape of the jets and their speed) is good.
\end{abstract}

\section{Lntroduction}

Dans une charge creuse classique équipée d'un revêtement métallique, il existe une vitesse limite de la pointe du jet qui ne peut être dépassée /1/. Cette vitesse, proportionnelle à la vitesse du son dans le matériau de revêtement, est par exemple de l'ordre de $13 \mathrm{~km} / \mathrm{s}$ pour un jet d'aluminium, et de $10 \mathrm{~km} / \mathrm{s}$ pour un jet de cuivre $/ 2 /$. Pour améliorer ces performances, il faut donc recourir à d'autres configurations de charges.

Nous proposons dans cette étude un nouveau système générateur de jets permettant d'atteindre dans certains cas, des vitesses supérieures à $20 \mathrm{~km} / \mathrm{s}$. Le principe consiste à attaquer par une onde de choc une cible évidée, et à profiter de la conjonction de deux effets :

- la mise en vitesse de la matière au fond de la cavité ;

- limplosion de la paroie de cette cavité.

Aprés avoir décrit les expériences effectuées, nous analysons, à l'aide de calculs numériques, les phénomènes liés à la formation et à la propagation du jet.

\section{Les expériences}

\section{Le dispositif expérimental}

Le dispositif expérimental conçu pour générer des jets hypervéloces (figure 1 - a) comporte un cylindre d'explosif dans lequel on initie une onde de détonation plane. Cette onde sert à mettre en vitesse un disque mince de cuivre qui vient impacter une cible métallique dans laquelle sont percées une ou plusieurs cavités cylindriques non débouchantes, caractérisées par leur diamètre $\phi$, leur profondeur h et le rayon de raccordement en fond de cavité $\mathrm{r}$ (égal à $\phi / 2$ dans le cas d'une cavité à fond hémisphérique). Le jet se forme au fond de la cavité, à l'arrivée de l'onde de compression engendrée par l'impact. 
Une variante de ce montage consiste à mettre l'explosif au contact de la cible ; celle-ci est donc directement attaquée par l'onde de détonation (figure 1 - b). On s'affranchit alors des problèmes de planété du choc transmis, dus à une éventuelle rotation de la plaque de cuivre.

Pour observer la propagation du jet formé, on utilise une méthode de cinématographie par ombre, en disposant à l'arrière et à proximité de ce jet un ou plusieurs flashs à argon. Des repères dimensionnels localisés sur la face avant du flash permettent de réaliser des mesures de vitesse.

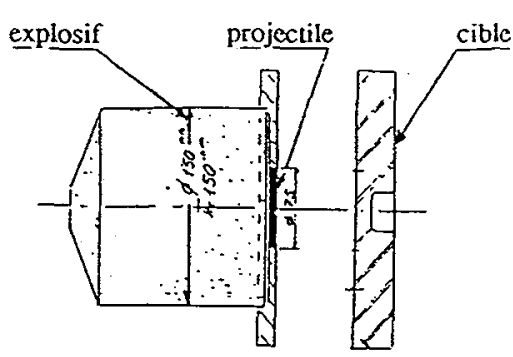

a-projection d'une plaque de cuive

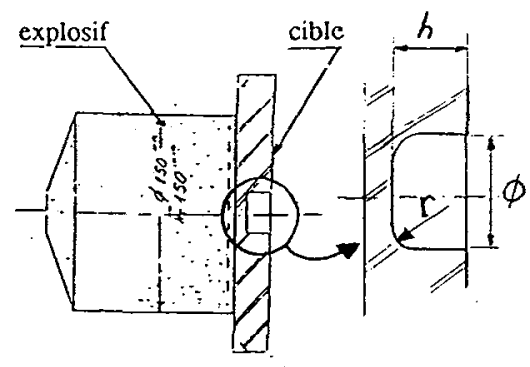

b-attaque directe par l'explosif

Fiqure 1 - Schéma du dispositif expérimental de création de jets hypervéloces

\section{Les résultats expérimentaux}

Un grand nombre d’expériences ont été réalisées en paramétrant la nature du matériau cible (cuivre, alliage d'aluminium AU4G), et la forme de la cavité : son diamètre $\phi$ (de 10 à $80 \mathrm{~mm}$ ), sa profondeur h (de 3 à $90 \mathrm{~mm}$ ) et le rayon de raccordement en fond de cavité $\mathrm{r}$ (de $\phi / 2$ à $\approx 0$ ). On constate qu'avec ce système, il est possible d'atteindre des vitesses de jet trés élevées, et cela d'autant plus que r est petit. Ainsi, avec une cible en AU4G à fond hémisphérique ( $\phi 20 \mathrm{~mm}, \mathrm{~h} 20 \mathrm{~mm}, \mathrm{r} 10$ $\mathrm{mm}$ ) on atteint tout juste $9.7 \mathrm{~km} / \mathrm{s}$; pour une cible de même nature, mais à trés faible rayon de raccordement $(\phi 20 \mathrm{~mm}$, h 20 $\mathrm{mm}, \mathrm{r} 1 \mathrm{~mm}$ ) on obtient $21,5 \mathrm{~km} / \mathrm{s}$ en tête de jet (figure 2). Pour une cible de cuivrè $\phi 30 \mathrm{~mm}, \mathrm{~h} 30 \mathrm{~mm}$, la vitesse est de $5,3 \mathrm{~km} / \mathrm{s}$ pour $\mathrm{r}=15 \mathrm{~mm}$, et de $15,5 \mathrm{~km} / \mathrm{s}$ pour $\mathrm{r}=3 \mathrm{~mm}$.

Par ailleurs, on constate une grande différence dans la forme du jet selon que la cavité a ou non un fond hémisphérique (figure 2). Dans le premier cas (figure 2 - a), on observe un jet relativement épais, parfois surmonté d'une protubérance (c'est surtout le cas avec lAU4G) ; par contre, les jets issus de cavités "à fond plat" sont constitués de trois zones distinctes (figures 2 - b et 2 - c) : on retrouve à l'arrière la partie épaisse du jet, couronnée par un panache se déployant progressivement, et dont la vitesse est de l'ordre de celle observée avec une cavité à fond hémisphérique ; à l'avant, on constate l'existence d'un dard trés mince qui constime le jet hypervelloce proprement dit.

Les jets ainsi formés sont suffisamment cohérents pour percer quelques dizaines de centimètres d'acier à 1 mètre de distance.

\section{Les simulations numériques}

Pour traiter correctement les phénomènes liés à la création, puis à la propagation des jets, il est nếcessaire d'utiliser des codes disposant d'équations d'état et de lois de comportement pouvant décrire à la fois le jet proprement dit (où la température est suffisante pour fondre et vaporiser partiellement le métal, et pour lequel un comportement hydrodynamique peut suffire), et la "périphérie" de la cavitê, où l'on doit prendre en compte l'aspect élastoplastique des matériaux. Deux codes ont été utilisés : PLEXUS et CEL.

\section{Le Code PLEXUS}

C'est l'un des codes éléments finis du système CASTEM /3/. Il peut traiter les géométries bi- ou tridimensionnelles. Son schéma explicite en temps le rend apte à traiter les problèmes de dynamique rapide et les grandes déformations. Deux équations d'état y sont disponibles : une équation "gaz parfait" et l'équation d'état de Wilkins, déjà utilisée pour la simulation des 
impacts à hypervitesse /4/. Les lois de comportement disponibles dans PLEXUS sont variées : hydrodynamique, élasto-
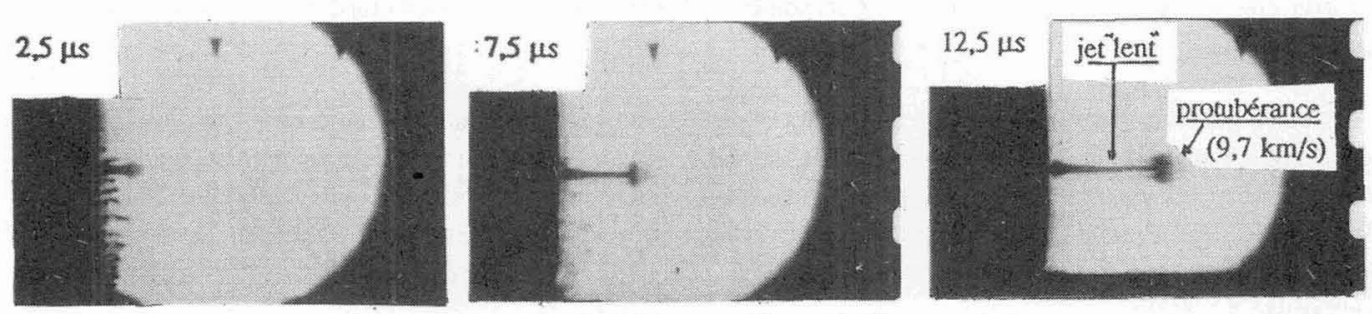

a-Cavité à fond hémisphérique - @ $20 \mathrm{~mm}, \mathrm{~h} 20 \mathrm{~mm}, \mathrm{r} 10 \mathrm{~mm}$
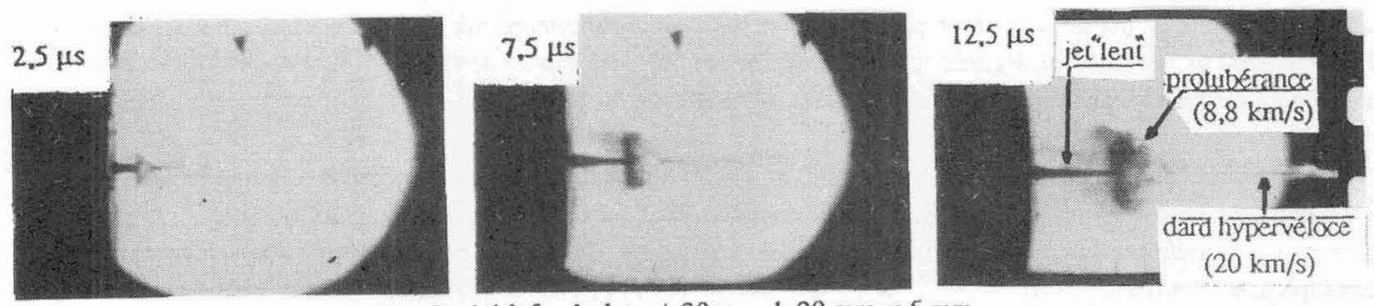

b- Cavité à fond plat - $020 \mathrm{~mm}, \mathrm{~h} 20 \mathrm{~mm}, \mathrm{r} 5 \mathrm{~mm}$

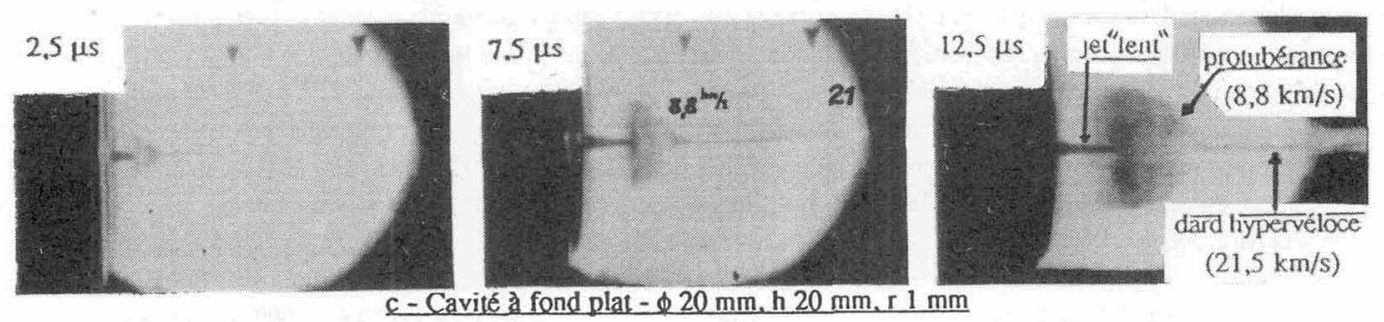

Figure 2-Visualisation par ombrographie des jets formés (cibles en AU4G)

(temps zero = sortie du jet de la cavité)

plastique avec ou sans écrouissage, et, éventuellement, prise en compte de l'influence de la température et de la vitesse de déformation (modèle de STEINBERG-COCHRAN-GUINAN en particulier $/ 5 /$ ).

\section{Le Code CEL}

Ce code bidimensionnel aux différences finies couplé Euler-Lagrange est plus riche en équations d'êtat que PLEXUS (en particulier il permet d'utiliser les équations SESAME) ; il dispose en outre de la loi de comportement dynamique de STENBERG-COCHRAN-GUINAN. Il a été utilisé dans des problèmes ciassiques d'impacts à hypervitesse $/ 4 /$, où il a permis de bien corréler les résultats expérimentaux.

Nous avons comparé ces deux codes pour différents types de cibles ; CEL a été testé en lagrangien et en eulérien. Les résultats obtenus sont trés voisins ; par exemple, avec une cible d'AU4G de cavité $\phi 20 \mathrm{~mm}$, h $20 \mathrm{~mm}, \mathrm{r} 1 \mathrm{~mm}$, PLEXUS calcule une vitesse maximale de jet de $21 \mathrm{~km} / \mathrm{s}$ et CEL (en lagrangien comme en eulérien), de $23 \mathrm{~km} / \mathrm{s}$. Pour une cible de cuivre de cavité $\phi 30 \mathrm{~mm}, \mathrm{~h} 30 \mathrm{~mm}, \mathrm{r} 3 \mathrm{~mm}$, on trouve $13 \mathrm{~km} / \mathrm{s}$ avec PLEXUS et $13,5 \mathrm{~km} / \mathrm{s}$ avec CEL.

\section{Phénoménologie de la crétion des jets}

Pour des cible en AU4G à cavité "à fond plat" ( $\phi 20 \mathrm{~mm}$, h $20 \mathrm{~mm}, \mathrm{r} 1$ à $5 \mathrm{~mm}$ ), nous avons étudié avec PLEXUS et CEL la formation du jet. La figure 3 montre la déformation progressive de la cible au cours du temps.

Compte tenu de la célérité du son dans l'AU4G $(\approx 6 \mathrm{~km} / \mathrm{s})$, l'onde de choc initiale débouche en fond de cavité vers $1,6 \mu$ s a à 
partir de $2 \mu$ s, on constate un relèvement de ce fond à la périphérie, là où s'amorce la courbure : il s'agit d'un effet classique de type "charge creuse", dû à la superposition de deux phénomènes: la projection vers l'avant du fond de la cavité, et la détente des paroies latérales.

Ceci explique que la vitesse de la "protubćrance" ainsi formée a une composante longitudinale (de lordre de 3,5 à $4 \mathrm{~km} / \mathrm{s}$ ) et une composante radiale convergente nettement plus faible, mais tendant à créer une accumulation de matière, donc une augmentation de la pression. D'autre part, sous l'effet du choc direct, le fond de la cavité s'accélère progressivement; sa vitesse est d'environ $10 \mathrm{~km} / \mathrm{s}$ à l'instant $(\approx 5 \mu \mathrm{s})$ où l'onde de compression centripète se focalise sur l'axe. On conçoit que le jet qui en résulte puisse atteindre des vitesses trés élevées, puisque ce second effet de charge creuse est engendré dans de la matière lancée à grande vitesse.

A partir de cet instant, on assiste à un développement rapide des mailles concentrées sur l'axe. La vitesse de tête du jet atteint $\approx 20 \mathrm{~km} / \mathrm{s}$ en $200 \mathrm{~ns}$, puis décroît légèrement, tandis que la protubérance initiale tend à se déployer, du fait des détentes radiales (figure 4) : ces résultats sont en accord avec l'expérience (figures 2 - b et 2 - c).

A linverse, pour une cavité à fond hémisphérique $(020 \mathrm{~mm}$, h $20 \mathrm{~mm}, \mathrm{r} 10 \mathrm{~mm})$, l'effet de charge creuse initial apparaît en fond de cavité sur l'axe de la cible ; la phase de focalisation est donc inexistante, et l'on ne pourra observer ni la surcompression ni, par conséquent, la création d'un dard hypervéloce (figure 4). Là encore, on retrouve qualitativement les observations expérimentales (figure 2 - a).

Des tracés d'isobares, d'isothermes et de courbes d'iso-densité à divers instants ont permis de préciser la phénoménologie de la formation et la constitution du jet. En particulier, on constate (figure 5) que, dans le cas de cavités à fond plat, la partie "hypervéloce" du jet n'a pas la même constitution que la partie située en arrière de la protubérance :

- la partie arrière (dont la vitesse est de l'ordre de $9 \mathrm{~km} / \mathrm{s}$ ) semble trés homogène ; la densité au voisinage de l'axe est proche de la densité initiale, pour des températures de lordre de $1000^{\circ} \mathrm{C}$ : cette partie du jet est donc liquide. Ces caractéristiques sont semblables à celles des jets obtenus avec une cavité à fond hémisphérique ;

- la partie avant est beaucoup plus hétérogène; elle se compose d'une zone liquide trés fine (le dard mis en évidence dans les expériences), de température voisine de celle de l'arrière, mais dont la densité, plus faible, décroît constamment le long du jet, et d'une zone gazeuse diffuse trés détendue et chaude $\left(2000\right.$ à $\left.3000^{\circ} \mathrm{C}\right)$ à la périphérie du dard liquide ; cette zone n'est pas visible dans les expériences sur AU4G, mais a pu être observée pour des jets de cuivre.
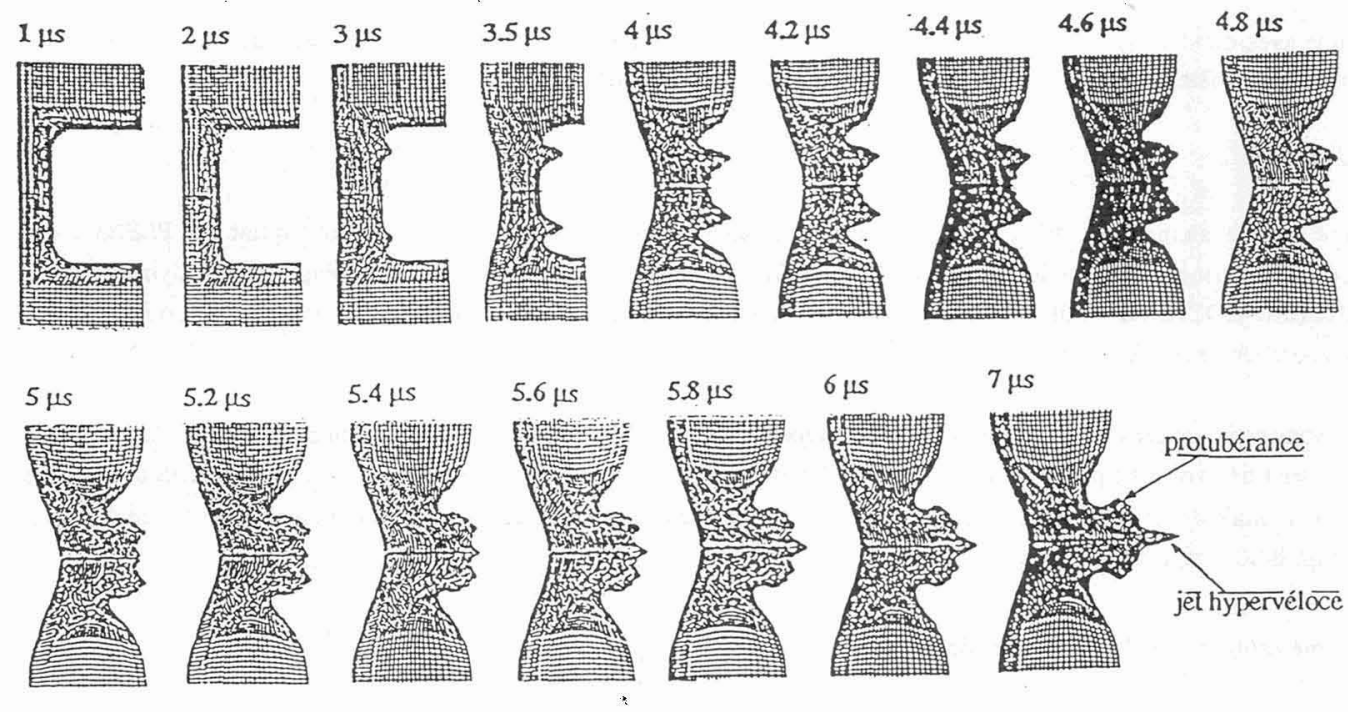

\section{Figure 3 - Formation du jet - Calcul effectue avec PLEXUS \\ Cible en AU4G. $\$ 20 \mathrm{~mm}$, h $20 \mathrm{~mm}, \mathrm{r} 5 \mathrm{~mm}$}

(les temps sont comptés à paririr de l'instant d'impact) 

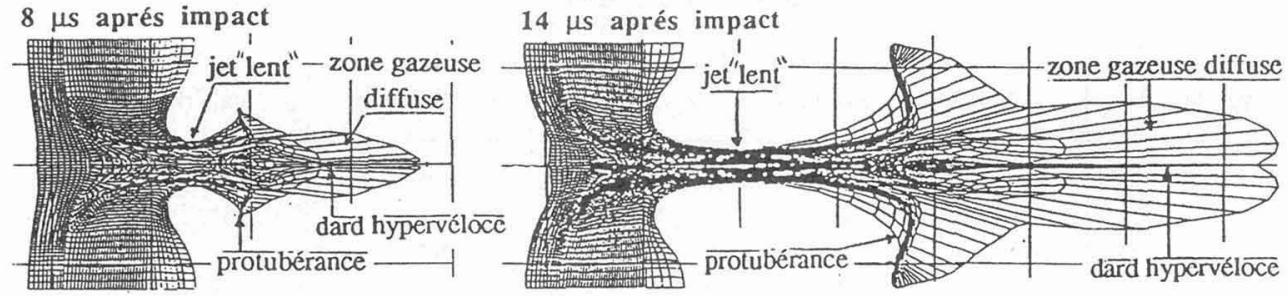

a-Cavité à fond plat $-20 \mathrm{~mm}, \mathrm{~h} 20 \mathrm{~mm}, \mathrm{I} 1 \mathrm{~mm}$
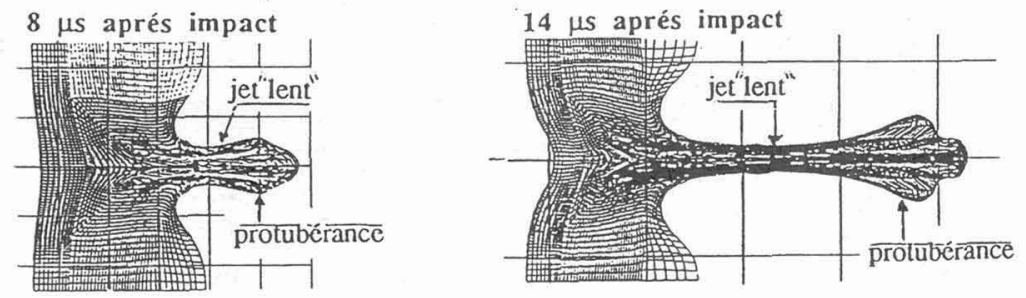

éch. : $2 \mathrm{~cm}$

\section{b-Cavité à fond hémisphérique $-\$ 20 \mathrm{~mm}, \mathrm{~h} 20 \mathrm{~mm}, \mathrm{r} 10 \mathrm{~mm}$}

Figure 4 - comparaison entre les jets issus de cavités à fond plat et à fond hémisphérique

Calcul effectué avec CEL - Cible en AU4G
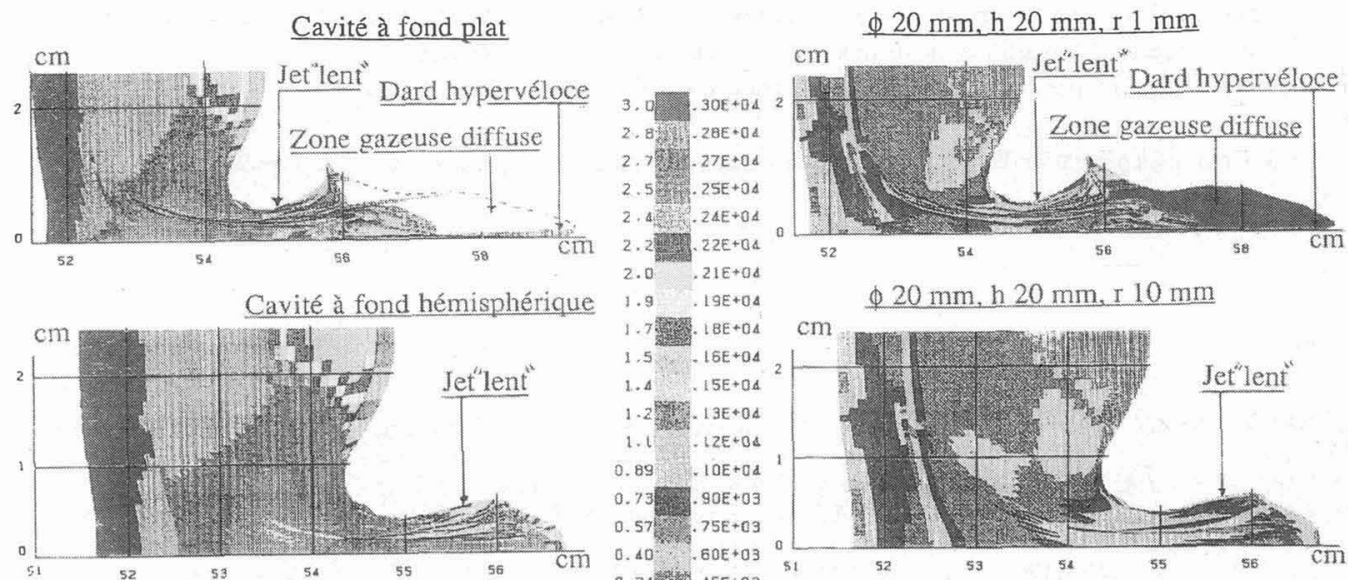

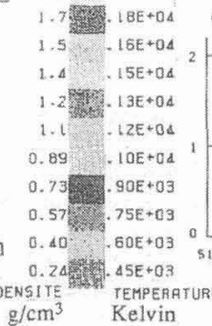

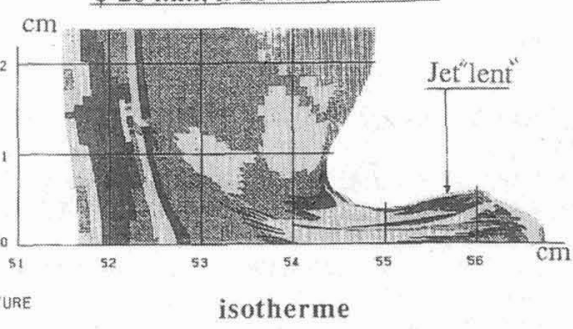

iso-densité

Figure 5 - Comparaison des isothermes et des courbes d'iso-densité

$8 \mu$ s aprés impact

Calculs effectués avec CEL - Cible en AU4G

\section{Comparaison expériences-calculs et conclusion}

Le tableau 1 présente un bilan des comparaisons expériences-calculs; la concordance est assez bonne. Toutefois, on constate que, pour des cibles en cuivre, le calcul tend à sous-évaluer la vitesse du jet, alors que c'est l'inverse pour des cibles en AU4G. En fait, les écarts les plus importants s'observent toujours quand la cible est directement attaquée par l'explosif ; dans ce cas, les conditions initiales du calcul (loi de pression sur la face arrière de la cible) sont connues avec une moins bonne précision que dans le cas d'un impact par projection de plaque. 
Tableau 1 - Comparaison expériences-calculs

* calcul CEL; ** calcul PLEXUS

\begin{tabular}{|c|c|c|c|c|c|}
\hline Type de cible & $\phi(\mathrm{mm})$ & $\mathrm{h}(\mathrm{mm})$ & $\mathrm{r}(\mathrm{mm})$ & $V \exp (\mathrm{km} / \mathrm{s})$ & $V$ calc $(\mathrm{km} / \mathrm{s})$ \\
\hline A U4G & 20 & 20 & 1 & 21,5 & $23^{*}, 21^{* *}$ \\
& 20 & 20 & 5 & 20 & $20^{*}, 18^{* *}$ \\
& 20 & 20 & 10 & 9,7 & $11,6^{*}$ \\
& 30 & 30 & 15 & 9,3 & $11,6^{*}$ \\
\hline Cuivre & 20 & 20 & 1 & 19 & $15,5^{*}$ \\
& 20 & 20 & 5 & 14,5 & $13,5^{* *}, 13^{* *}$ \\
& 30 & 30 & 3 & 5,5 & $5^{*}$ \\
\hline
\end{tabular}

Il reste que les études numériques ont permis de confirmer l'ensemble des observations expérimentales, et d'expliquer la formation de jets hypervéloces. De nouvelles expériences et calculs sont en cours pour maîtriser totalement les phénomènes d'éjection à trés haute vitesse. Ils ont en particulier pour but :

- d'améliorer la compréhension du processus d'alimentation du jet, en particulier dans sa partie hypervéloce;

- d'évaluer la quantité de matière autour de la cavité qui participe à l'éjection ;

- d'étudier finement la "qualit $e$ " du jet (gradient de vitesse le long du jet, élongation maximale avant rupture...) ;

- de préciser linfluence de certains paramètres : type d'onde de détonation (plane, sphérique divergente ou conique), caractéristiques élastoplastiques du matériau cible (notamment sa courbe d'écrouissage) :

- d'étudier d'autres types de cibles (multicouches par exemple), et d'autres formes de cavité.

Ces travaux doivent nous permettre d'optimiser les performances cinétiques des jets ainsi obtenus et d'améliorer leur pouvoir perforant.

\section{Références}

III M. DEFOURNEAU - "Sciences et Techniques de l'armement"- Mémorial de l'artillerié française - Tome 44 - 2eme fascicule - 1970.

12I W.P. WALTER - J.A. ZUKAS - Fondamentals of shaped charges - John Wiley and Sons - 1989.

131 M. LEPAREUX - H. BUNG - Système CASTEM - Programme PLEXUS - Notice d'utilisation - Note CENS/ DEMT/LAMS du 13 AOUt 1987.

14I J.P. LEYRAT - E. CHARVET - M. LACOMME - P. MASSARD - H.C. PUJOLS - Simulation des effets des impacts à hypervitesse - Communication présentée au DYMAT 91.

15I DJ. STEINBERG - S.G. COCHRAN - M.W. GUINAN - A constitutive model applicable at high strain rate-Journal of applied physics - 51 (3) - Mars 1980.

161 W.H. GUST - High impact deformation of metal cylinders at elevated temperatures - Journal of applied physics - 53 (5)-pages 3566-3575-1982. 